\title{
A New Realization of the Global Delaunay-Voronoi Tesselation in Arbitrary Dimension*
}

\author{
R. Riedinger ${ }^{1,2}$, M. Habar ${ }^{2}$, P. Oelhafen' ${ }^{1}$ and H.J. Güntherodt ' . \\ 1 Institut für Physik. Universität Basel. Klingelbergstrasse 82, CH 4056 BASEL \\ 2 Lab.de Pnysique et de Spectroscopie Electronique. Université de Haute Alsace. \\ F 68093 MULHOUSE CEDEX.
}

The dual Delaunay-Voronoi tesselations provide an unambiguous description of the topological structure of random media and a covering of space around the generating points. Numerous algorithms have been proposed for the voronoi tesselation and more recently, for the dual Delaunay one. We present a property characterizing simplices of all intermediate dimension constituting Delaunay cells which allows the full construction of the Delaunay tesselation, by recurrence over the dimension of the space. We derive by duality, without calculation, the full dual Voronoi tesselation from a tree of parentship over the Delaunay tiles, which may be restricted to adjacent dimensions.

The paper is presented as a dialog, in the style of Hume-Rothery's book /1/. Candide, a newcomer in the field of liquid and amorphous materials, discusses with his host Mentor.

C: You suggested me to examine several works /2-5/. I am puzzled by the fact that if one wants to characterize the state of order of the matter beyond the pair correlation function, the dual tesselations of Voronoi and Delaunay seem to be the best tools available ;wy a limited number of physicists only is able to perform tris analysis?

$M$ : It is true that the definition of the two tesselations is quite simple, but their general and effective construction is up to now hard to realize. A Voronoi cell is a convex polyhedron, the generalization of a wigner-Seitz cell

* Presented at the Sixth International Conference on Liquid and Amorphous Metals, Garmisch-Partenkirchen, FRG, August 24 to 29, 1986. 
to random media. A point $x$ belongs to the voronoi cell $v i$ attached to $x i$, if $x$ is closer to $x i$ than to any other vertex $\times j / 6-8 /$, ( $f i g 1$ )

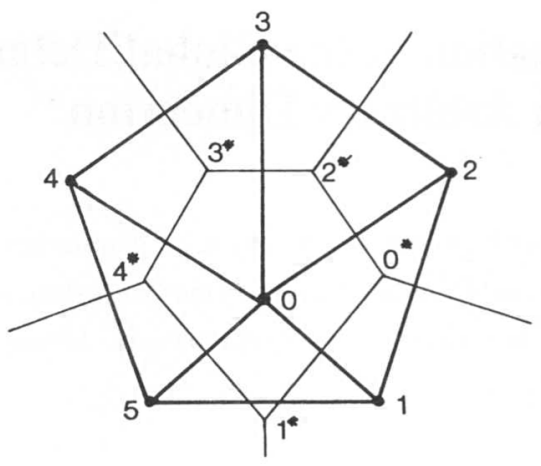

Fig 1 : A set of "random points (labelled 0-5) is the squeleton of the Delaunay tiling (thick line); the dual Voronoi tiling is represented in thin lines Labels for all elements can be found in table 1 .

C : I prefer to some extend the dual Delaunay tesselation $/ 9 /$. because the cells are simpler than in the Voronoi one : triangles, tetrahedra in dim. 2 and 3 resp.: one might describe them by a set of $(d+1)$ points in a $d-d i m$. space (a simplex), (i1 i2 id+1), which one might even order lexicographically i1< i2< $\ldots<i d+1$. I find the property that characterizes a Delaunay tile nice :

There falls no other vertex but the generating ones, inside the circumsphere to a tile: furthermore, the center of this sphere is the corresponding dual voronol vertex /6/.

I just generalized it for regular sub-tiles of lower dimension of the Delaunay tesselation /10/:

if no other point falls into the d-dim. circumsphere to a di-dim simplex

$0 \leq d i \leq d$, this simplex belongs to the Delaunay tesselation

This allows to build the Delaunay tesselation, by recurrence over the dimension, by adjoining a further point to an existing tile and checking its regularity (fig 2 ).

$M$ : It seems to me that you miss some segments in this construction: for instance. $\left(\begin{array}{ll}1 & 5\end{array}\right)$ does not verify your regularity criterion.

C: $(15)$ is indeed irregular ; but I obtain it as a part of a nigher dim. tile $(015)$. One has to be patient, and to perform a downward recurrence over dimension to complete the list of tiles. 


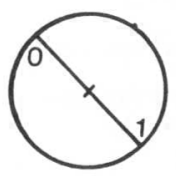

a

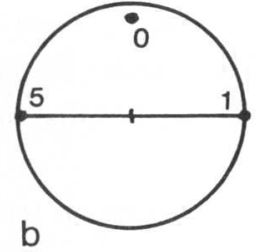

b

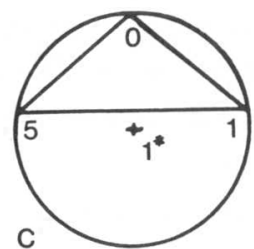

Fig 2 : a) A regular tile $(1: 0)$; b) a non regular tile $(1: 6)$; c) $(1: 6)$, alias (1.5) belongs to the $(2: 1)$ tile obtained from $(1: 0)$ by adjoining (5). Note that the center of the circumsphere is [1].

$M$ : We get indeed a whole hierarchy of tiles. ordered over dimension and inside each dimension. ( $d i: i)$ designates a unique $t i l e$. We report them in table 2 . One is tempted to glue the constituting pieces together, to precise the structure : for this. we can define a tree of chiloren :

$(d i+1: j)$ is a son of $(d i: i)$ if $(d i: i)$ is contained in it There exists also a tree of parents ! But let us complete table 1 by the children.

$C$ : At the end of our Delaunay construction, we obtained the Voronoi dual vertex of each simplex (di:i), as the center of the circumsphere. I label these points as $\left(d: i^{*}=[0: i]=[i]=i^{*}\right.$. How could one obtain the whole Voronoi tesselation now?

$M$ : It is well known that the dual $[1: i\}$ to the nyperplane containing $(d-1: i)$ is the segment bounded by the Voronoi vertices of cells twinned by this face. But these points are related to the sons of $(d-1: i)$. How could we precise and generalize this property?

C : It seems first that duality makes correspond elements of complementary dimension in each tesselation :

$(d i: i)<--->[d-d i: i]$

Then, we may describe a Voronoi tile through its boundary

$[d i: i]=[[0 i-1: i 1] \ldots[d i-1: i j]]$

This means that $[0 i: i]$ is the convex cell bounded by $[0 i-1: i 1] \ldots$

Such a description exists in Rodgers /11/. The dual of [di:1] is (d-di:i): we take its sons. $(d-d i+1: i 1) \ldots(d-d i+1: i j)$, and dua $i$ back !

So, we can build all dual elements by recurrence over the dimension also, and complete table 2 upwards.

$M$ : We have just a problem with the unpaired outer cells at the boundary, like [1:5] ; it may be easily overcomed by specifying that $[1: 5]$ is the outward half-line originating from $[0: 0]$ orthogonal to $(1: 5)$

$[1: 5]=[[0](1: 5)]$ 
We get really the whole structured dual tesselation of efther Delaunay or Voronoi tesselation by inspection only, without having to build convex cells from a convex hull of points! Perhaps is this that mathematicians call duality of tesselations?

$C$ : It can be even realized by hand on the simplest cases; it provides a global. non sequential approach, independent of the dimension of space and of the boundaries. Every body should be able now to perform polyhedron analysis !

$M$ : Well, let us hurry to come back to physics and to apply it ourselves to our problems. Where have you put the sticks and balls to build models for our materials?

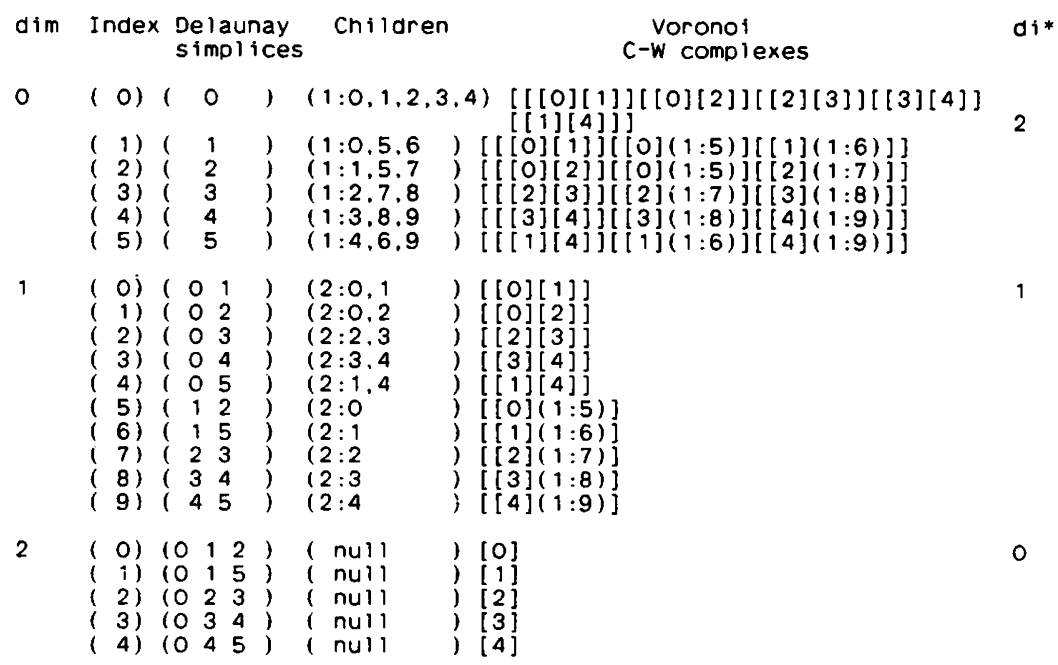

Table I: Delaunay tesselation, $(c 01,1,2,3)$ is realized by a recurrence upwards over the dimension, then downwards to obtain the non regular tiles. Dual Voronoi vertices are obtained at this stage. The children are build by inspection. The Voronoi cells are then built upwards. The index is shared by both tesselations.

\section{Acknowledgments.}

R. Riedinger whishes to thank to his colleagues at Basel for their friendly hospitality. Calculations have been performed at URZ Basel and at CIRCE in APL 


\section{References}

$/ 1 /$ w. Hume-Rothery, "An introduction to metallurgy", London, Inst itute of Metal,

/2/Ed. F. Yonezawa and T. Ninomiya, "Topological disorder in condensed matter", Berlin, Springer, 1983.

/3/ R. Collins, in "Phase transitions and critical phenomena" vol 2 ed $C$. Domb and M.S. Green, New-York, Academic press, 1972, ch 7 p 271-303.

/4/T. Ogawa, M. Tanemura, Progr. Theor. Phys. 51,399 (1982)

/5/D.R. Nelson, Phys.Rev. B28,5515 (1985)

/6/ J.L. Finney, Journ. of Computat.Physics 32,137 (1979)

/7/W. Borstow,J.P. Dussault and B.L. Fox, J.of Computat. Phys.2.819 (1978)

/8/ J.M. Augenbaum and C.S. Peskin, J.of Computat. Priys.59, 177 (1985)

/4/T. Ogawa, M. Tanemura, Progr. Theor. Phys. 51,399 (1982)

/9/ M. Tanemura,T.Ogawa and N. Ogita, J.of Computat.Phys. 51,191 (1983)

$/ 10 /$ R. Riedinger.M. Habar,P. Oelnafen and H.J. Güntherodt.J.of Computat. Phys. . submitted.

/11/ C.A. Rodgers "Packing and covering" , London, Cambridge Univ. Press, 1964 